Utah State University

DigitalCommons@USU

\title{
Examining a Mediation Model of Body Image-Related Cognitive Fusion, Intuitive Eating, and Eating Disorder Symptom Severity in a Clinical Sample
}

Jennifer L. Barney

Utah State University, jen.barney@usu.edu

Tyson S. Barrett

Utah State University, tyson.barrett@usu.edu

Tera Lensegrav-Benson

Avalon Hills Eating Disorder Specialists

Benita Quakenbush

Avalon Hills Eating Disorder Specialists

Michael P. Twohig

Utah State University, michael.twohig@usu.edu

Follow this and additional works at: https://digitalcommons.usu.edu/psych_stures

Part of the Psychology Commons

\section{Recommended Citation}

Barney, J.L., Barrett, T.S., Lensegrav-Benson, T., Quakenbush, B., Twohig, M.P. (in press). Examining a mediation model of body image-related cognitive fusion, intuitive eating, and eating disorder symptom severity in a clinical sample. Eating and Weight Disorders - Studies on Anorexia, Bulimia, and Obesity

This Article is brought to you for free and open access by the Psychology Student Works at DigitalCommons@USU. It has been accepted for inclusion in Psychology Student Research by an authorized administrator of DigitalCommons@USU. For more information, please contact digitalcommons@usu.edu.

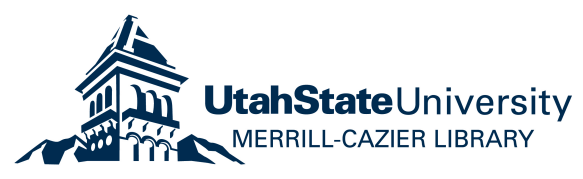




\section{Examining a Mediation Model of Body Image-Related Cognitive Fusion, Intuitive Eating, and Eating Disorder Symptom Severity in a Clinical Sample}

Jennifer L. Barney, M.S. a, Tyson S. Barrett, Ph.D. ${ }^{\text {a }, ~ T e r a ~ L e n s e g r a v-B e n s o n, ~ P h . D . ~}{ }^{\text {, }}$ Benita Quakenbush, Ph.D. ${ }^{\mathrm{b}}$, Michael P. Twohig, Ph.D. ${ }^{\mathrm{a}}$

${ }^{a}$ Department of Psychology, Utah State University 2810 Old Main Hill, Logan UT 84322 USA

${ }^{\mathrm{b}}$ Avalon Hills Eating Disorder Specialists, 175 E 100 N, Logan, UT 84321 USA

Corresponding author:

Jennifer L. Barney

Department of Psychology

Utah State University

2810 Old Main Hill

Logan, UT 84322

Office: (435) 797-8303

Email: jen.barney@usu.edu 


\begin{abstract}
Purpose: This study sought to explore the associations between Intuitive Eating (IE), eating disorder (ED) symptom severity, and body image-related cognitive fusion within a clinical sample. IE was also examined as a possible mediator in the relationship between body imagerelated fusion and ED symptoms.

Methods: This study includes cross-sectional analyses with data from 100 adult females and 75 adolescent females seeking residential treatment for an ED. Self-reported demographic information, ED symptoms, IE behaviors, and body image-related cognitive fusion were collected from participants within the first week of treatment following admission to the same residential ED treatment facility.
\end{abstract}

Results: ED symptom severity was significantly negatively associated with three of the four domains of IE; unconditional permission to eat, reliance on hunger and satiety cues, and bodyfood choice congruence. A significant mediational effect of IE on the relationship between body image-related fusion and ED symptoms through IE behaviors was observed $(\beta=11.3, \mathrm{SE}=.003$, $p<.001)$. This effect was only observed for the unconditional permission to eat $(\beta=.13, p=.003)$ and reliance on hunger and satiety cues $(\beta=.10, p=.005)$ domains of IE when the domains were subsequently analyzed individually.

Conclusion: Unconditional permission to eat and reliance on hunger and satiety cues appear to be particularly influential domains of IE in the relationship between body image-related fusion and ED symptom severity. It is possible that changes in these IE domains may be mechanisms through which body image-related fusion influences ED symptoms. Future longitudinal research is needed to better understand the relationship between body image-related cognitive fusion and IE and the potential for targeting these constructs specifically in the context of ED treatment. 
Keywords: Eating Disorders, Intuitive Eating, Cognitive Fusion, Body Image, Mediation Analysis

Level of Evidence: Level V, cross-sectional analysis from descriptive study 


\section{Declarations}

\section{Funding}

No funds, grants, or alternative financial supports were received to conduct this study.

\section{Conflicts of Interest/Competing Interests}

The authors have no conflicts of interest or competing interests to declare.

\section{Data Availability}

The dataset analyzed for this study is not publicly available, however the data may be provided from the corresponding author upon reasonable request.

\section{Code Availability}

The statistical code used for analyses in this study is available from the corresponding author upon reasonable request.

\section{Ethics Approval}

All questionnaires and methodology for the current study were approved by the Utah State University Institutional Review Board [Ref \#]. All procedures used in the study adhered to the tenets of the Declaration of Helsinki.

\section{Consent to Participate}

Prior informed consent was obtained from all participants (and parents for all adolescent participants). The consent forms were examined and approved by the above Institutional Review Board.

\section{Consent for Publication}

Informed consent for publication of analyses using the obtained data was included in the aforementioned written consents. 
Eating disorders (EDs) are serious, sometimes fatal conditions characterized by a persistent disturbance of eating-related behaviors (e.g. restriction; bingeing; purging) that significantly impair one's physical health and/or psychosocial functioning [1]. Individuals with EDs also frequently experience a range of body image disturbances including dissatisfaction, preoccupation, and overvaluation of weight and shape [2]. Etiological and maintenance models posit that engagement in dieting and exercise behaviors when primarily motivated by desires to changes one's weight/shape is a risk factor for the development of disordered eating pathology [3]. Empirical studies exploring this relationship have consistently found internalization of the thin ideal, social pressures to be thin, history of dieting, weight suppression, and body image disturbance are all significantly predictive of future ED onset [4]. Similarly, rigid dietary control (i.e. inflexible, dichotomized "all or nothing" dietary rules dictating what, when, and how one should eat) has reliably been linked with disordered eating $[5,6]$.

Such findings suggest that strategies minimizing dietary control motivated by external cues and promoting more adaptive and sustainable patterns of eating may be particularly useful for the treatment of EDs. Intuitive eating (IE) is one such approach characterized by a strong connection with and understanding of internal physiological hunger and satiety cues when determining eating behaviors to adequately meet physical and emotional needs [7]. The authors of the IE approach discuss how humans are innately born with the wisdom and ability to integrate instinct, emotion, and thought in the ways necessary to facilitate IE behaviors. However, as humans grow older, they develop more advanced cognitive abilities and encounter diverse social contexts (e.g. familial, cultural, media) which often promote different messages surrounding food and body. The complex integration of these psychosocial factors into the individual's eating behaviors transitions them from primarily instinctual, to a dynamic interplay 
between instinct, thoughts, feelings, and perceived social norms [7]. Therefore, simply having these abilities does not guarantee successful IE. For many, over time accessing these abilities becomes increasingly difficult due to lack of practice and/or internalization of conflicting messages from various external environments which can cause significant deviations from the adaptive eating behaviors IE describes. The IE framework therefore strives to increase the adaptive use of these abilities and remove potential barriers to doing so [7].

Tribole \& Resch outline ten principles of IE which guide individuals in how to build and practice IE within their own lives [7]. These principles have been clustered into four core theoretical domains that together contribute to overall levels of engagement in IE. The first domain, Unconditional Permission to Eat, refers to how willing an individual is to eat when they experience physical hunger and to refuse categorical labels such as "good" or "bad," "allowed" or "forbidden," in relation to specific foods. The second domain, Reliance on Hunger and Satiety Cues, refers to an individuals' trust in their internal bodily cues of hunger or satiety and how much they rely on these cues to guide their eating behaviors. Third, the Eating for Physical Rather Than Emotional Reasons domain refers to an individual's reasoning for choosing to engage in patterns of eating behavior in which they are eating to satisfy physical hunger and meet their bodies nutritional needs and/or eating to cope with emotional distress. Finally, the fourth domain, Body-Food Choice Congruence, refers to the extent individuals match their food choices to align with their bodies' nutritional needs to promote healthy physical functioning. The first three domains were included as individual subscales within the original Intuitive Eating Scale developed to measure IE as a latent construct [8], and more recently a revised version of the measure, the Intuitive Eating Scale - 2 (IES-2), was created to incorporate the additional Body-Food Choice Congruence domain [8]. 
A breadth of research exists indicating that IE is inversely related to levels of disordered eating symptoms as well as many ED risk factors including internalization of the thin ideal, body dissatisfaction, body shame, and negative affect $[9,10]$. A recent meta-analysis of IE in relation to psychological correlates found that IE was significantly and negatively associated with bingepurge symptoms, eating restraint, emotional eating, global eating pathology, internalization of appearance ideals, and shape and weight concerns [10]. Among the 97 studies included, only four examined IE behaviors among individuals with clinically significant eating disorders, and all four compared these samples to healthy controls. Healthy controls consistently reported significantly higher levels of intuitive eating compared to individuals with eating disorders, with a large pooled effect size [9]. Similarly, Van Dyck, Herbert [11] found that women with an ED had significantly lower scores on all IES-2 subscales compared to woman without an ED diagnosis. Further, the authors found that when looking at individual ED diagnoses, women with binge eating disorder had higher scores on the Unconditional Permission to Eat subscale when compared to women with anorexia nervosa or bulimia nervosa, and that women diagnosed with anorexia nervosa had higher scores on the eating for physical rather than emotional reasons subscale compared to those with bulimia nervosa or binge eating disorder. Research also suggests that at least some components of intuitive eating are associated with various indices of well-being above and beyond the variance accounted for by low ED symptomology [12]. Further, the absence of ED symptomology does not always predict IE/adaptive eating behaviors $[12,13]$. These findings suggest that IE and ED symptomology are distinct but related constructs and provide initial support for investigating IE as a potential intervention target for individuals with EDs. 
Despite the consistent finding that IE behaviors are inversely related to ED symptoms across clinical and non-clinical samples, there is little research to date exploring changes in IE in relation to ED symptoms over time, and even less examining the incorporation of IE practices when treating individuals with EDs. In a prospective study of IE behaviors among young adults, individuals characterized as "intuitive eaters" at time one reported lower levels of unhealthy weight control behaviors (i.e. fasting, using meal substitutes, skipping meals, smoking cigarettes, using diet pills, diuretics, or laxatives, and/or making oneself vomit in a desire for weight loss) and lower rates of binge eating accompanied by loss of control at five year follow up [14]. Notably however, individuals were categorized as "intuitive eaters" in this study based only on their responses to two questions taken from the original Intuitive Eating Scale (i.e. 'I stop eating when I feel full' and 'I trust my body to tell me how much to eat'). In another prospective study among a sample of 1,270 women, higher baseline levels of intuitive eating predicted lower odds of onset for eight ED related symptoms (i.e. objective binge eating, subjective binge eating, purging, driven exercise, fasting, overvaluation of weight and shape, and fear of weight gain) at 8-month follow up [15]. Further, intuitive eating uniquely predicted lower odds of subjective binge eating or purging onset above and beyond those predicted by body appreciation and selfcompassion [14]. In a two-year pilot study evaluating an inpatient/residential eating disorder treatment program focused on the development of intuitive eating practices, participants had significant increases in patient's IE behaviors from pre- to post-treatment. This study also found that when different ED diagnoses were considered, individuals diagnosed with bulimia nervosa reported the lowest levels of IE at baseline but experienced the greatest improvement in IE from pre- to post-treatment whereas individuals diagnosed with anorexia nervosa or eating disorder not otherwise specified reported higher levels of IE compared to those with a bulimia nervosa 
diagnoses at baseline and experienced only moderate improvements in this regard. Finally, in a study examining the presence of adaptive eating among individuals in recovery from an eating disorder, findings indicated that individuals who were in full recovery from their eating disorder (defined as absence of a current ED diagnoses, BMI $\geq 18.5 \mathrm{~kg} / \mathrm{m} 2$, absence of any binge eating, purging, or fasting behaviors within the previous 3 months, and scores within 1 SD of agematched community norms on measures of ED related cognitions) did not differ from healthy controls on overall levels of intuitive eating or any IE domains. These individuals also reported significantly greater levels of total IE behaviors, Unconditional Permission to Eat, and Reliance on Hunger and Satiety Cues when compared to individuals in partial recovery or currently diagnosed with a clinically significant ED, and significantly greater levels of Eating for Physical Rather than Emotional Reasons compared to individuals with a current ED.

While these findings are promising, significant gaps in the literature exist that are essential for informing future interventions for EDs that target increases in IE behavior. First, much of the research examining the relationship between IE and ED symptomology examines IE as a unidimensional construct and does not consider the four theoretically and empirically supported domains of IE individually. Understanding the unique role each domain may play in the development and maintenance of disordered eating behaviors among those with clinically significant EDs is essential to inform the development of effective and efficient interventions targeting IE for individuals with EDs. Second, the vast majority of research exploring the relationship between IE and ED symptoms to date has been conducted using college students and/or non-clinical community samples. While this literature provides initial insights as to how variations in IE levels may relate to elevated ED symptomology, empirical replication of these findings within clinically significant samples is needed in order to generalize this knowledge and 
appropriately inform clinical intervention. The current study therefore seeks to address these gaps by examining the unique relationship between each of the four IE domains and ED symptom severity within a clinically significant ED sample.

The research denoting the inverse relationship between IE and ED symptomology also presents an important question for additional research: what are the most important barriers impeding the adaptive use of one's intuition, thoughts, and feelings that may explain why some individuals engage in lower levels of IE and, in turn, develop higher levels of ED pathology? Understanding the mechanisms through which this relationship occurs in this way is vital for identifying therapeutic processes that may be most beneficial to target in IE interventions for those with EDs. Further, identification of these mechanisms would provide insight as to when and why certain instances of lower IE behavior are indicative of elevated ED pathology and others are not. One process that may theoretically play a role in the inverse relationship between IE and ED in this way is body image-related cognitive fusion. Body image-related cognitive fusion is defined as the entanglement with the verbal content of ones' thoughts related to body image, interpreting them as complete truth and acting accordingly, rather than recognizing them as simply thoughts representing only one interpretation of reality [16]. Recently, theoretical models of ED psychopathology have been developed that posit body image-related cognitive fusion may be a core maintenance mechanism of ED symptoms [17, 18]. These models propose that due to high levels of body image-related fusion, individuals utilize unhealthy eating and weight-related behaviors associated with EDs (e.g. dietary restriction; binging and/or purging; over-exercise) in an effort to alleviate their distressing body image-related thoughts without consideration of health or values-based contingencies. 
High levels of body image-related cognitive fusion are consistently associated with poorer psychological health and quality of life [19] as well as higher levels of ED symptomology $[20,21]$. Previous path analysis research has found that body image-related cognitive fusion appears to play a significant role in explaining inflexible eating patterns among young women [22]. Further, body image-related fusion was found to be a key process in explaining the links between several known ED risk factors and self-reported ED symptoms among female college students [16]. Based on these findings, we propose that body image-related cognitive fusion may indirectly influence ED symptom severity through its effect on IE behaviors. In other words, we hypothesize that increases in body image-related cognitive fusion explain decreased engagement in IE behaviors, and that this relationship subsequently influences one's experience of elevated ED symptoms.

The current study seeks to expand upon previous knowledge regarding the inverse relationship between IE and ED symptoms by: 1) examining the unique associations between each of the four IE domains and ED symptom severity in a clinically significant ED sample, 2) examine IE as a potential mediator in the relationship between body image-related cognitive fusion and ED symptom severity, and 3) if IE is observed as a mediator in this relationship, test a parallel mediation model to explore whether certain domains of IE appear to drive this mediating effect. Because lower levels of IE, higher levels of body image-related cognitive fusion, and higher levels of ED symptom severity are all commonly associated with lower psychological well-being and higher of depression- and anxiety-related symptoms, we also sought to examine whether the observed relationships between each variable remained significant even after variance explained by levels of depression and anxiety symptoms was considered. Based on previous findings, we predict that lower scores on the unconditional permission to eat, reliance 
on hunger and satiety cues, and eating for physical rather than emotional reasons domains of IE would all be significantly associated with elevated ED symptom severity and higher levels of body image-related fusion. With regard to the body food choice congruence domain of IE, we predict that lower body food choice congruence would be associated with higher body imagerelated fusion, but not higher ED symptom severity based on previous findings that this domain tends to be linked more closely to body-related variables as opposed to ED symptomology [11, 23]. We further predict that the relationship between body image-related fusion and ED symptom severity will be mediated by IE behaviors. Due to the exploratory nature of our analyses considering each IE domain independently, we did not have any specific a priori hypotheses about whether all or only some of the IE domains would drive this mediating effect.

\section{Participants}

\section{Methods}

All data were collected at a for-profit residential treatment facility located in the Western U.S. for adolescent (ages 11-17) and adult (aged 18 and older) females struggling with eating disorders, where the average length of stay for clients is between 4 and 6 months. The current study included 175 females consecutively admitted to either the adolescent $(n=75)$ or adult $(n=$ 100) residential treatment unit between November 2015 and June 2020. All participants were diagnosed with an eating disorder as defined by the Diagnostic and Statistical Manual of Mental Disorders-Fifth Edition [1]. Descriptive statistics for the sample are presented in Table 1.

\section{Procedures}

All parts of the study were approved by a university institutional review board. Participant recruitment was conducted via convenience sampling where, throughout the data collection period, every client admitting to the residential treatment facility (and parents or legal 
guardians of adolescent clients) was informed about the opportunity to participate in research and given details regarding what participation would entail. All were informed that participation in the study was voluntary and that their choice to participate or not would have no impact on the clinic treatment they would receive while at the residential facility. A total of 17 individuals and/or parents opted not to participate in the study. Clients who provided informed consent (and assent in the case of adolescents) completed an online self-report assessment battery within the first three days following their intake. The assessment battery included demographic information, assessment of ED symptom severity, and a variety of assessment measures related to comorbid psychopathology, therapeutic processes, and targeted treatment outcomes.

\section{Measures}

\section{Eating Disorder Examination Questionnaire [EDE-Q; 24]}

The EDE-Q was utilized to assess eating disorder symptom severity and includes 28 selfreport items that assesses core attitudinal features and behaviors indicative of eating disorder psychopathology. Each item is answered on a 7-point Likert scale $(0=$ No days; $6=$ Everyday $)$ indicating how often the respondent engaged in a disordered eating behavior or experienced an eating disorder related cognition over the last 28 days. Due to researcher error, item 8 ("In the past 28 days how has thinking about shape or weight made it very difficult to concentrate on things you are interested in?) and item 25 ("In the past 28 days how dissatisfied have you been with your weight?) were unintentionally omitted. However, missing data on the EDE-Q is notoriously common [25] and guidelines for handling missing data provided by the authors of the EDE-Q indicate that valid totals can still be computed for the EDE-Q if a majority of the items are completed [24]. In the current study, Cronbach's alpha for the EDE-Q was .92.

\section{Cognitive Fusion Questionnaire-Body Image [CFQ-BI; 26]}


The CFQ-BI is a 10-item self-report measure designed to assess cognitive fusion related to body image. Responses are rated on a 7-point Likert scale $(1=$ Never True; $7=$ Always True $)$ to indicate how frequently each item (e.g. "I get so caught up in my thoughts about my physical appearance that I am unable to do the things that I most want to") applies to the individual. The CFQ-BI has been previously validated in both clinical [27] and non-clinical samples [26] and has been found to have good incremental validity relative to general measures of cognitive fusion, as well as excellent internal consistency (Cronbach's alpha $=.96)$. Internal consistency of the CFQBI was also excellent within the current study (Cronbach's alpha $=.97)$.

\section{Intuitive Eating Scale-2 [IES-2; 23]}

The IES-2 measures individuals' tendency to attend to their physical hunger and satiety cues and utilize these cues to determine when, what, and how much to eat. The IES-2 consists of 23 self-report items with responses rated on a 5-point likert scale $(1=$ Strongly Disagree, $5=$ Strongly Agree) with regard to one's attitudes and behaviors. Higher scores indicate higher levels of intuitive eating. Internal consistency for the total scale in the current sample was excellent $(\alpha=.92)$. The IES-2 also produces four subscale scores: Unconditional Permission to Eat $(\alpha=.89)$, Reliance on Hunger and Satiety Cues $(\alpha=.92)$, Eating for Physical rather than Emotional Reasons $(\alpha=.94)$, and Body-Food Choice Congruence $(\alpha=.85)$.

\section{Beck Depression Inventory, $2^{\text {nd }}$ Edition [BDI-II; 28]}

The BDI-II is a widely used measure of depression severity and is composed of 21 -items related to various affective, cognitive, and physical symptoms frequently associated with depression. Items are rated from 0-3 with regard to severity, and a total score (0-63) is calculated by summing item scores such that higher scores indicate greater levels of depression. 
The psychometric properties of the BDI-II are well established among [29], including in clinical eating disorder samples [30]. Internal consistency in the current sample was excellent $(\alpha=.91)$.

\section{Beck Anxiety Inventory [BAI; 31]}

The BAI is a widely used self-report measure of anxiety symptom severity composed of 21 items rated on a 4 -point likert scale $(0=$ not at all to $3=$ severely $)$ with regard to how distressing symptoms had been during the past month. The psychometric properties of the BAI are well established $[31,32]$ and the BAI demonstrated excellent internal consistency in the current sample $(\alpha=.91)$.

\section{Data Analysis}

All analyses were conducted with $\mathrm{R}$ in RStudio, version 3.5.2 [33, 34]. The psych [35] package was utilized to examine assumptions; the jmv [36] package was utilized to calculate descriptive statistics and bivariate correlations; and the lavaan package [37] was used for the mediation analyses. Missing data was addressed using the full information maximum likelihood (FIML) estimation function in the lavaan package, which is recommended as the most pragmatic approach to missing data estimation for SEM analyses and has been demonstrated to produce unbiased parameter estimates and standard errors [38]. Bivariate relationships between ED symptom severity, body image-related fusion, the four domains of intuitive eating, depression symptoms, and anxiety symptoms were examined first using Pearson correlations. We then conducted a mediation analysis to test the prediction that IE scores would mediate the relationship between body image-related cognitive fusion and ED symptom severity. Based on the results of these analyses, we then used parallel mediation analysis to examine which of the four domains of intuitive eating appeared to contribute to the hypothesized mediated relationship, and to determine whether any of the domains appeared to drive the mediation more 
than others. Bootstrapping with 5000 bootstrapped samples was used to calculate $95 \%$ confidence intervals for all indirect effects. All coefficients and indirect effect results are presented in standardized units. In all analyses, age, BMI, depression symptoms, and anxiety symptoms were included as control variables. In an effort to control for potential differences based on ED diagnosis despite the large differences in sample sizes for each diagnosis, we created a dichotomous variable categorizing participants into two groups based on ED behavioral profile consistencies between diagnoses and included inclusion in either group as an additional control variable. Specifically, we included all participants diagnosed with either Anorexia Nervosa-Restrictive Subtype or Avoidant and Restrictive Food Intake Disorder (i.e., diagnoses characterized by restrictive behaviors) in group 1, and all participants diagnosed with Anorexia Nervosa - Binge/Purge Subtype, Bulimia Nervosa, or Binge Eating Disorder (i.e., diagnoses characterized by engagement in binging and/or purging behaviors). The two individuals diagnosed with Other Specified Feeding or Eating Disorder were not included in either group due to having no access to the clinical data used regarding these individuals' behavioral profiles to determine this diagnosis. This categorization method is consistent with those used in previous research [e.g., 27, 39].

\section{Results}

Means and standard deviations on each measure as well as the results of the bivariate correlation analyses are presented in Table 2. Notably, when compared to the three non-clinical female samples included in the validation study of the IES-2 [23], the average total IE score within the current sample was $>1$ standard deviation lower (indicating less IE behavior). At the domain level however, the mean level of eating for physical rather than emotional reasons and body-food choice congruence reported in the current sample was consistent with the mean 
reported in all three non-clinical female samples. Conversely, the mean scores in both the unconditional permission to eat domain and the reliance on hunger and satiety cues domain were both $>1.5$ standard deviations below those reported in the validation studies by Tylka \& Van Diest [23].

Higher levels of ED symptom severity were positively associated with higher body image-related fusion scores, and negatively associated with higher levels of unconditional permission to eat, reliance on hunger and satiety cues, and body-food choice congruence. Surprisingly, no association was found between ED symptom severity and the eating for physical rather than emotional reasons IE domain. Higher levels of body image-related cognitive fusion were significantly negatively associated with scores on all IE domain subscales, indicating that individuals who were more fused with their body image-related thoughts reported less engagement in all four domains of IE behavior.

To test the prediction that IE behaviors mediate the relationship between body imagerelated cognitive fusion and ED symptom severity, we conducted a simple mediation model using total IE scores as the mediator. Consistent with our hypothesis, the indirect effect of body image-related cognitive fusion on ED symptom severity through IE behaviors was significant $(\beta=.11, S E=.003, p<.001)$. In the total model, age $(\beta=.08, S E=.007, p=.040)$, BMI $(\beta=-.09$, $S E=.015, p=.029)$, and anxiety $(\beta=.16, S E=.008, p=.009)$ were all significant individual predictors of ED symptom severity. In other words, higher age, lower BMI, and higher anxiety were significantly predictive of higher ED symptom severity. Only age $(\beta=-.21, S E=.005$, $p=.001)$ and ED behavioral profile $(\beta=.14, S E=.094, p=.042)$ were significant predictors of the mediator (total IE scores) such that younger individuals and individuals with a restrictive ED behavior profile reported significantly higher levels of IE. 
The finding that total IE scores did mediate the relationship between body image-related fusion and ED symptom severity supported additional parallel mediation analyses examining each of the domains of IE independently as mediators (Figure 1). The results of these analyses revealed that body image related cognitive fusion is indirectly related to ED symptom severity through its relationship with both the unconditional permission to eat and reliance on hunger and satiety cues domains of IE. Specifically, body image-related cognitive fusion had a significant, negative direct effect on reported levels of unconditional permission to eat ( $\mathrm{a}_{1}$ path) and unconditional permission to eat scores had a significant, negative direct effect on ED symptom severity after controlling for the effect of body image-related fusion ( $b_{1}$ path). Similarly, body image-related cognitive fusion had a significant, negative direct effect on reported levels of reliance on hunger and satiety cues (a2 path), and lower reliance on these cues had a significant, negative direct effect on ED symptom severity after controlling for the effect of body imagerelated fusion ( $b_{2}$ path). The indirect effect of body image-related cognitive fusion on ED symptom severity through unconditional permission to eat was statistically significant $(\beta=.14$, $p=.001, \mathrm{ci}=.007$ to .024$)$. The indirect effect of body image-related cognitive fusion on ED symptom severity through reliance on hunger and satiety cues was also statistically significant $(\beta=.08, p=.004, \mathrm{ci}=.003$ to .015$)$.

In contrast, neither of the direct effects of body image-related cognitive fusion on the eating for physical rather than emotional reasons (az path) or body-food choice congruence ( $a_{4}$ path) domains of IE were statistically significant. Similarly, neither the direct effect of eating for physical rather than emotional reasons on ED symptom severity ( $b_{3}$ path) or the direct effect of body food choice congruence on ED symptom severity $\left(b_{4}\right)$ after controlling for the effects of body image-related fusion were statistically significant. Finally, the indirect effects of body 
image-related fusion on ED symptom severity through the eating for physical rather than emotional reasons domain $(\beta=-.004, p=.573, \mathrm{ci}=-.002$ to .001$)$ and the body food choice congruence domain $(\beta=-.000, p=.970$, ci=-.001 to .001$)$ were both not statistically significant.

With regard to covariates included in the total model anxiety scores $(\beta=.11, S E=.007$, $p=.036)$, and ED behavioral profile $(\beta=-.15, S E=.126, p<.001)$ were both significant predictors of ED symptom severity whereas age $(\beta=.06, S E=.006, p=.062)$, $\operatorname{BMI}(\beta=.04, S E=.014, p=.254)$ and depression scores $(\beta=.096, S E=.008, p=.132)$ were not. In other words, higher anxiety scores and having a diagnosis characterized by a binge and/or purge behavioral profile were significantly predictive of higher level of ED symptom severity within the model. When examining the covariates predictive significance of each mediator (i.e. each individual IE domain) within the model, age was only a statistically significant predictor of scores on the Reliance on Hunger and Satiety Cues IE domain $(\beta=-.22, S E=.008, p=.001)$, depression scores were only a significant predictor of scores on the Body-Food Choice Congruence IE domain $(\beta=-.29, S E=.009, p=.015)$, and ED behavioral profile was only a significant predictor of scores on the Eating for Physical Rather than Emotional Reasons IE domain $(\beta=.32, S E=.183, p<.001)$. No other covariates were significant individual predictors of the four mediators within the model.

Overall, body image-related cognitive fusion had a significant direct effect on ED symptom severity, even when taking into account the indirect effects of body image-related fusion through all four domains of intuitive eating (c' path). Further, the total effect of body image-related cognitive fusion on ED symptom severity was significant $(\beta=.61, \mathrm{SE}=.007$, $p<.001)$ and the mediation model as a whole explained $78.9 \%$ of the total variance in ED symptom severity.

\section{Discussion}


Overall, the results of our study indicate that females diagnosed with an ED seeking residential treatment report lower overall levels of IE compared to non-clinical female samples, and that at the domain level this decrease appears to be due to lower reported levels of unconditional permission to eat and lower reliance on hunger and satiety cues. ED symptom severity was significantly, negatively associated with all but the eating for physical rather than emotional reasons domain of IE. A significant indirect effect of body image-related cognitive fusion on ED symptom severity through IE behaviors was also observed and the unconditional permission to eat and reliance on hunger and satiety cues domains of IE appear to drive this mediation effect.

The non-significant association between the eating for physical rather than emotional reasons IE domain and ED symptom severity is somewhat inconsistent with previous literature that has found significant associations between the eating for physical rather than emotional reasons domain of IE and ED symptoms in non-clinical samples [e.g., 23, 40]. One possible explanation for this finding may be due to the high proportion of individuals diagnosed with Anorexia-Restrictive subtype within our sample. Prior research examining emotional eating behaviors across eating disorder diagnoses has found that individuals with Anorexia Nervosa tend to report eating less than usual (i.e. engaging in restrictive eating behaviors) in response to negative emotions, particularly among those with an AN-restrictive type diagnosis [41, 42]. Because the eating for physical rather than emotional reasons subscale of the IES-2 only assesses one's tendency to use food as a coping mechanism for emotional distress as a result of negative affect [23], it is possible that this subscale does not capture the ways in which individuals with Anorexia Nervosa diagnoses use food as an emotional coping mechanism. This is further supported by our finding that individuals with an eating disorder diagnosis categorized by binge 
and/or purge behaviors reported significantly lower levels of IE within this domain. Future research should therefore seek to examine the relationship between the individual IE domains and ED symptom severity in more diverse diagnostic samples, and specifically examine potential differences across diagnostic profiles.

The statistically significant association observed between the body-food choice congruence domain of IE and ED symptom severity was contrary to our prediction, although this correlation was comparatively much smaller than those observed between ED symptom severity and the unconditional permission to eat and reliance on hunger and satiety cues domains. This subscale was added as a core domain of IE more recently than the other three IE domains and has consequently been less well-explored [13]. While previous research has found little to no association between scores on the body-food choice congruence subscale and ED symptomology $[11,23]$ there are notable differences between the samples used in these studies relative to ours that may account for these differences. First, the samples in these studies were made up of college students/community members as opposed to those currently experiencing a clinically significant eating disorder. Therefore, it is possible that a general lack of ED symptomology, or differences in the way the items making up the body-food choice congruence subscale are interpreted by those with clinically significant EDs, may have prevented a significant relationship from being observed. Specifically, the three items included in the body-food choice congruence subscale focus on a desire to eat "nutritious foods" most of the time, typically eating foods perceived to give one's body "stamina or energy" and to eat foods that "make one's body perform well." Many individuals with EDs report elevated levels of orthorexic behavior [43, 44], where a focus on only consuming "healthy" or "nutritious" foods becomes a persistent fixation and/or alters eating behavior to an extreme where avoidance of foods considered 
"unhealthy" leads to an overly-restricted diet. It is therefore possible that the items of the bodyfood choice congruence subscale may be highly endorsed by individuals who are experiencing orthorexia symptoms, despite the pathological aspects of these food choices which are inconsistent with the adaptive strategies promoted by IE. Future research should explore the overlap and validity of this subscale in adequately assessing IE among individuals experiencing orthorexia or eating disorder pathology in this way.

Further, previous samples were made up of male and female community members, whereas the current sample included only females. It is therefore possible that gender differences moderate the relationship between body-image food congruence and ED symptom severity. In fact, Van Dyck, Herbert [11] found that scores on the body-food choice congruence scale of the IES-2 were significantly different between males and females, and found that among females body-food choice congruence was significantly associated with various behavioral ED risk factors, whereas among men these scores were only significantly associated with body image-related factors. While specific conclusions cannot be made based solely on the current findings, future research should seek to examine the role of body-food choice congruence in relation to ED symptoms among both male and female individuals with clinically significant ED symptoms to better understand the nature of this relationship.

Findings also revealed that the body image-related relationship between body imagerelated fusion and ED symptom severity appears to be mediated by the unconditional permission to eat and reliance on hunger and satiety cues domains on IE, but not the eating for physical rather than emotional reasons or body-food choice congruence domains. Unconditional permission to eat incorporates the willingness to eat when hungry and to eat foods one enjoys and desires and encourages an "emotionally neutral" stance toward all foods. Individuals who 
experience a high level of body image-related cognitive fusion may become "fused" to emotionally charged thoughts or messages they have received about foods' impact on their body (e.g. "chocolate causes bloating' or "avoiding sugar will result in weight loss") such that these thoughts drive their eating behaviors without consideration of their preferences or dietary needs. Similarly, reliance on hunger and satiety cues requires a willingness to let one's own visceral signals of hunger and fullness dictate when and how much one chooses to eat. However, if an individual is highly fused to thoughts such as "someone my height and weight should only eat 1500 calories or less a day," they may rigidly adhere to this rule regardless of whether or not it is in line with their bodily signals. As body image-related cognitive fusion begins to affect the unconditional permission to eat and reliance on hunger and satiety cues domains of IE in this way, our findings suggest that among individuals with clinically diagnosed EDs, the severity of their ED symptoms increases. These findings are consistent with previous research indicating that a history of dieting and attempts to follow rigid food rules are associated with elevated ED symptoms $[45,46]$ and the importance of "rejecting the diet mentality" emphasized as the first principle for adopting an IE framework [7], and suggest that these two IE domains may be particularly important when considering future interventions for EDs.

Our findings provide initial support for the theory that interventions targeting increases in IE behaviors, particularly unconditional permission to eat and reliance on hunger and satiety cues may be beneficial for women struggling with EDs. Our study also suggests that these IE domains may be particularly impacted by elevated levels of body image-related cognitive fusion among individuals with EDs, and that this predicts increased ED severity. These findings are encouraging for future exploration of interventions directly targeting body image-related cognitive fusion for individuals with EDs to determine whether they might be useful for 
promoting increased engagement in adaptive IE behaviors, especially unconditional permission to eat and reliance on hunger and satiety cues to guide eating behavior. However, it is important to note the cross-sectional nature of the relationships observed in the current study, and longitudinal replication demonstrating whether this pattern is sustained over time is needed.

Recent research examining the use of interventions such as Acceptance and Commitment Therapy (ACT), which specifically focus on decreasing one's fusion with distressing thoughts and feelings, for individuals with EDs have been found to be effective in reducing ED symptoms and body image distress $[47,48]$. A core therapeutic process in ACT is cognitive defusion, which seeks to remove unhelpful attachments to one's thoughts and increase one's ability to mindfully observe them with dispassionate curiosity [49]. While defusing from one's thoughts related to body image alone may not influence one's eating behaviors, it may provide individuals with the ability to "step back" from these thoughts and choose their eating behaviors based on what "works" for their overall well-being, as opposed to acting primarily to alleviate or neutralize their thoughts. In this way, defusion interventions for individuals with EDs may provide individuals with strategies to distance themselves from distressing thoughts about their body while engaging in more adaptive IE behaviors regardless of the content of their thoughts at any given time. While theoretically, this process is in line with those posited by ACT interventions, longitudinal replication of our analyses and research specifically examining cognitive defusion as a process mechanism in interventions for EDs is needed to support this notion. To date, there is a small but growing body of evidence suggesting that ACT is effective in decreasing ED symptom severity and body image concerns [linardon et al 2017, 2019], and that among a sample of overweight/obese adults, led to significant improvements in the eating for physical rather than emotional reasons component of IE from pre- to post-intervention. 
However, to our knowledge, no studies have explicitly examined the use of ACT-based interventions targeting body image-related fusion within individuals with eating disorders, or examined this as a possible therapeutic mechanism of change that may promote adaptive eating behaviors such as IE within these populations.

\section{Limitations}

The findings from this study should be interpreted with some limitations in mind. First, the data utilized for analyses were cross-sectional, meaning longitudinal conclusions regarding the temporal nature of the observed mediation effects cannot be made. Future research should therefore seek to examine the proposed model in a longitudinal manner. Second, all individuals in our study were admitting to a residential level of care. Thus, replication of these findings is needed in clinical samples receiving lower level of care (e.g., day treatment programs; outpatient services) and non-treatment seeking samples to determine the generalizability of our results. Third, the relative homogeneity of our sample with regard to gender, ethnicity, and diagnoses limits the generalizability of our findings. While our sample is relatively consistent with the diagnostic composition commonly observed among residential ED samples, the low level of Bulimia Nervosa, Binge Eating Disorder, and Avoidant and Restrictive Food Intake Disorder diagnoses prevents us from drawing conclusions generalizable to these populations. Similarly, the majority of our clients identified as white females and resided in the United States, which limits the generalizability of our findings to male ED clients or individuals with EDs from nonwhite or non-westernized cultures.

\section{Conclusion}

In conclusion, our findings suggest that the unconditional permission to eat and reliance on hunger and satiety cues domains of IE are particularly influential in the relationship between 
body image-related cognitive fusion and ED symptom severity. Specifically, our study provides initial support for the theory that increases in body image-related fusion are related to decreased levels of unconditional permission to eat and reliance on hunger and satiety cues, and that this relationship, in part, predicts elevated ED symptoms. Based on these findings, future research examining these mediational effects longitudinally to determine whether changes in body imagerelated fusion appear to drive changes in IE, and consequently, changes in ED symptoms is warranted. Such research would provide further support for the possible benefits of using cognitive defusion interventions with one's body image-related thoughts to promote adaptive IE behaviors and/or reducing ED symptomology.

\section{What is already known on this subject?}

Consistent evidence denotes an inverse relationship between IE behaviors and ED symptoms, however much of the research to date has been conducted in non-clinical samples and examines IE as a singular construct. Further, theoretical models incorporating cognitive processes such as body image-related fusion that may influence one's engagement in various IE behaviors and/or experiences of ED symptoms have not yet been explored in an effort to better understand this relationship.

\section{What this study adds?}

This study highlights that each of the unique IE domains appears to be differentially related to ED symptom severity and suggests that unconditional permission to eat and reliance on hunger and satiety cues may be particularly relevant in predicting ED symptom severity. Further, these findings provide initial support for the theoretical notion that elevated levels of body image-related fusion predict decreases in these IE behaviors, and this effect, in turn predicts elevated ED symptom severity. These preliminary findings highlight the need for future 
longitudinal examination of this process and suggest body image-related cognitive fusion may be important to address in therapeutic interventions for EDs seeking to promote IE behaviors. 
Table 1. Descriptive Statistics

\begin{tabular}{lccc}
\hline & $\begin{array}{c}\text { Adolescents } \\
(\mathrm{n}=75)\end{array}$ & $\begin{array}{c}\text { Adults } \\
(\mathrm{n}=100)\end{array}$ & $\begin{array}{c}\text { All } \\
(\mathrm{N}=175)\end{array}$ \\
\hline Age $M(\mathrm{SD})$ & $15.17(1.47)$ & $26.01(8.21)$ & $21.47(8.29)$ \\
\hline BMI $M(\mathrm{SD})$ & $19.27(2.93)$ & $20.16(4.65)$ & $19.79(4.04)$ \\
Ethnicity $N(\%)$ & & & \\
White/Caucasian & $67(89.3)$ & $93(93.0)$ & $160(91.95)$ \\
Asian & $2(2.70)$ & $1(1.0)$ & $3(1.72)$ \\
Biracial & $5(6.76)$ & $5(5.0)$ & $10(5.75)$ \\
Native Hawaiian & $0(0)$ & $1(1.0)$ & $1(.57)$ \\
Diagnosis $N(\%)$ & & & \\
AN-R & $42(56.0)$ & $45(45.0)$ & $87(49.71)$ \\
AN-B/P & $24(32.0)$ & $38(38.0)$ & $62(35.43)$ \\
BN & $7(9.33)$ & $12(12.0)$ & $19(10.86)$ \\
BED & $1(1.33)$ & $2(2.0)$ & $3(1.71)$ \\
ARFID & $0(0)$ & $2(2.0)$ & $2(1.14)$ \\
OSFED & $1(1.33)$ & $1(1.0)$ & $2(1.14)$ \\
Years since ED & $2.73(1.70)$ & $11.62(9.12)$ & $7.87(8.28)$ \\
symptoms began & & & \\
\hline
\end{tabular}

Note: AN-R: Anorexia Nervosa-Restrictive Type; AN-B/P: Anorexia Nervosa-Binge/Purge Type; BN: Bulimia Nervosa; BED: Binge Eating Disorder; ARFID: Avoidant and Restrictive Food Intake Disorder; OSFED: Other Specified Feeding and Eating Disorder 
Table 2. Means, Standard Deviations, and Bivariate Correlations

\begin{tabular}{|c|c|c|c|c|c|c|c|c|c|c|}
\hline \multirow[b]{2}{*}{ Variable } & \multirow[b]{2}{*}{$M$} & \multirow[b]{2}{*}{$S D$} & \multicolumn{8}{|c|}{ Correlations } \\
\hline & & & 1 & 2 & 3 & 4 & 5 & 6 & 7 & 8 \\
\hline 1. EDE-Q & 3.94 & 1.56 & - & - & - & - & - & - & - & - \\
\hline 2. CFQ-BI & 52.70 & 15.08 & $.79 * * *$ & - & - & - & - & - & - & - \\
\hline 3. IES-Total & 2.87 & .70 & $-.64 * * *$ & $-.58 * * *$ & - & - & - & - & - & - \\
\hline 4. IES-UPE & 2.29 & 1.03 & $-.71 * * *$ & $-.57 * * *$ & $.59 * * *$ & - & - & - & - & - \\
\hline 5. IES-RHSC & 2.21 & 1.08 & $-.65 * * *$ & $-.56 * * *$ & $.82 * * *$ & $.61 * * *$ & - & - & - & - \\
\hline 6. IES-EPR & 3.60 & 1.14 & -.11 & $-.17 *$ & $.64 * * *$ & -.10 & $.23 * *$ & - & - & - \\
\hline 7. IES-BFCC & 3.37 & .99 & $-.19 *$ & $-.20 * *$ & $.41 * * *$ & .10 & $.29 * * *$ & .12 & - & - \\
\hline 8. BAI & 26.45 & 12.04 & $.52 * * *$ & $.46^{* * *}$ & $-.27 * * *$ & $-.37 * * *$ & $-.24 * *$ & -.02 & -.07 & - \\
\hline 9. BDI & 33.82 & 12.47 & $.65 * * *$ & $.66 * * *$ & $-.46 * * *$ & $-.48 * * *$ & $-.42 * * *$ & -.11 & $-.22 * *$ & $61 * * *$ \\
\hline
\end{tabular}
$* * * p<.001, * * p<.01, * p<.05$

Note: EDE-Q: Eating Disorder Examination Questionnaire Global Score; CFQ-BI: Cognitive Fusion Questionnaire-Body Image Total Score; IES-Total: Intuitive Eating Scale-2 Total Score; IES-UPE: Intuitive Eating Unconditional Permission to Eat Subscale Score; IES-RHSC: Intuitive Eating Reliance on Hunger and Satiety Cues Subscale Score; IES-EPR: Intuitive Eating Eating for Physical Rather than Emotional Reasons Subscale Score; IES-BFCC: Intuitive Eating Body-Food Choice Congruence Subscale Score; BAI: Beck Anxiety Inventory Score; BDI: Beck Depression Inventory-II Score 
Figure 2 Parallel mediation model illustrating unconditional permission to eat, reliance on hunger and satiety cues, eating for physical rather than emotional reasons, and body-food choice congruence as mediators of the relationship between body image-related fusion and ED symptom severity

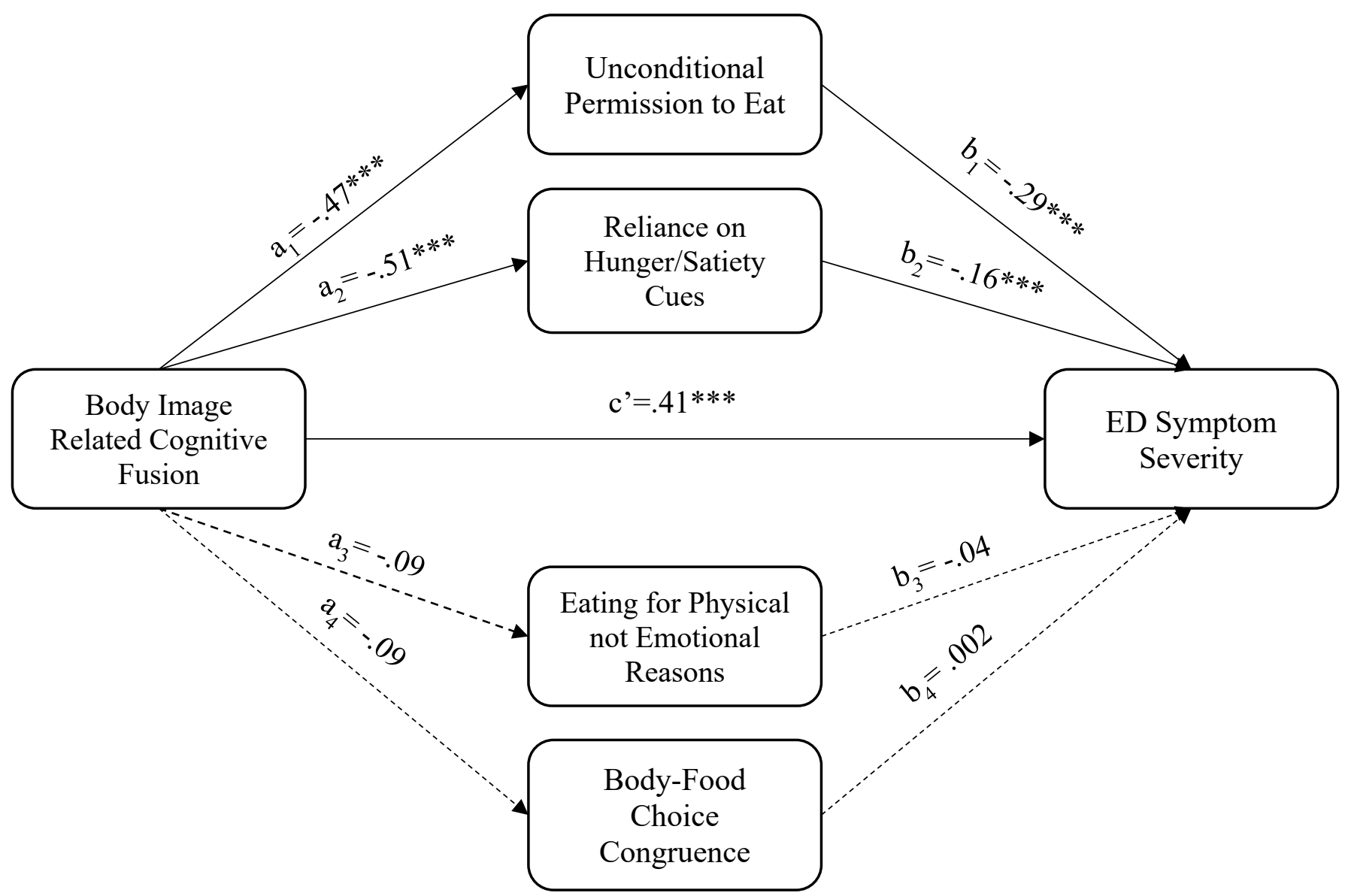

$* * * p<.001$

Note: All coefficients are standardized. 


\section{References}

1. American Psychiatric Association (2013) Diagnostic and statistical manual of mental disorders (DSM-5®). 5 ed. Arlington, VA.

2. Sharpe H, Griffiths S, Choo T H, et al. (2018) The relative importance of dissatisfaction, overvaluation and preoccupation with weight and shape for predicting onset of disordered eating behaviors and depressive symptoms over 15 years. International Journal of Eating Disorders 51(10):1168-1175. DOI: https://doi.org/10.1002/eat.22936.

3. Pennesi J-L and Wade T D (2016) A systematic review of the existing models of disordered eating: Do they inform the development of effective interventions? Clinical Psychology Review 43:175-192. DOI: https://doi.org/10.1016/j.cpr.2015.12.004.

4. Stice E (2016) Interactive and mediational etiologic models of eating disorder onset: Evidence from prospective studies. Annual Review of Clinical Psychology 12:359-381. DOI: https://doi.org/10.1146/annurev-clinpsy-021815-093317.

5. Westenhoefer J, Engel D, Holst C, et al. (2013) Cognitive and weight-related correlates of flexible and rigid restrained eating behaviour. Eating Behaviors 14(1):69-72. DOI: https://doi.org/10.1016/j.eatbeh.2012.10.015.

6. Linardon J and Mitchell S (2017) Rigid dietary control, flexible dietary control, and intuitive eating: Evidence for their differential relationship to disordered eating and body image concerns. Eating Behaviors 26:16-22. DOI: https://doi.org/10.1016/j.eatbeh.2017.01.008.

7. Tribole E and Resch E (2012) Intuitive eating. New York, NY: Macmillan. 
8. Hawks S, Merrill R M, and Madanat H N (2004) The intuitive eating scale: Development and preliminary validation. American Journal of Health Education 35(2):90-99. DOI: https://doi.org/10.1080/19325037.2004.10603615.

9. Bruce L J and Ricciardelli L A (2016) A systematic review of the psychosocial correlates of intuitive eating among adult women. Appetite 96:454-472. DOI: https://doi.org/10.1016/j.appet.2015.10.012.

10. Linardon J, Tylka T L, and Fuller-Tyszkiewicz M (2021) Intuitive eating and its psychological correlates: A meta-analysis. International Journal of Eating Disorders. DOI: https://doi.org/10.1002/eat.23509.

11. Van Dyck Z, Herbert B M, Happ C, Kleveman G V, and Vögele C (2016) German version of the intuitive eating scale: Psychometric evaluation and application to an eating disordered population. Appetite 105:798-807. DOI: https://doi.org/10.1016/j.appet.2016.07.019.

12. Tylka T L and Wilcox J A (2006) Are intuitive eating and eating disorder symptomatology opposite poles of the same construct? Journal of Counseling Psychology 53(4):474. DOI: https://doi.org/10.1037/0022-0167.53.4.474.

13. Cadena-Schlam L and López-Guimerà G (2015) Intuitive eating: An emerging approach to eating behavior. Nutrición Hospitalaria 31(3):995-1002. DOI: https://www.redalyc.org/articulo.oa? id=309235369001.

14. Christoph M, Järvelä-Reijonen E, Hooper L, et al. (2021) Longitudinal associations between intuitive eating and weight-related behaviors in a population-based sample of young adults. Appetite 160. DOI: https://doi.org/10.1016/j.appet.2021.105093. 
15. Linardon J (2021) Positive body image, intuitive eating, and self-compassion protect against the onset of the core symptoms of eating disorders: A prospective study. International Journal of Eating Disorders 54:1967-1977. DOI: https://doi.org/10.1002/eat.23623.

16. Trindade I A and Ferreira C (2014) The impact of body image-related cognitive fusion on eating psychopathology. Eating Behaviors 15(1):72-75. DOI: https://doi.org/10.1016/j.eatbeh.2013.10.014.

17. Merwin R M and Wilson K G (2009) Understanding and treating eating disorders: An ACT perspective. Acceptance and commitment therapy: Contemporary theory, research, and practice:87-117.

18. Manlick C F, Cochran S V, and Koon J (2013) Acceptance and commitment therapy for eating disorders: Rationale and literature review. Journal of Contemporary Psychotherapy 43(2):115-122. DOI: https://doi.org/10.1007/s10879-012-9223-7.

19. Ferreira C and Trindade I A (2015) Body image-related cognitive fusion as a main mediational process between body-related experiences and women's quality of life. Eating and Weight Disorders-Studies on Anorexia, Bulimia and Obesity 20(1):91-97. DOI: https://doi.org/10.1007/s40519-014-0155-y.

20. Ferreira C, Palmeira L, and Trindade I A (2014) Turning eating psychopathology risk factors into action. The pervasive effect of body image-related cognitive fusion. Appetite 80:137-142. DOI: https://doi.org/10.1016/j.appet.2014.05.019.

21. Duarte C, Pinto-Gouveia J, and Ferreira C (2017) Ashamed and fused with body image and eating: Binge eating as an avoidance strategy. Clinical Psychology \& Psychotherapy 24(1):195-202. DOI: https://doi.org/10.1002/cpp.1996. 
22. Trindade I A and Ferreira C (2015) Falling in the traps of your thoughts: The impact of body image-related cognitive fusion on inflexible eating. Eating Behaviors 19:49-52. DOI: $\underline{\text { https://doi.org/10.1016/j.eatbeh.2015.06.004. }}$

23. Tylka T L and Kroon Van Diest A M (2013) The Intuitive Eating Scale-2: Item refinement and psychometric evaluation with college women and men. Journal of Counseling Psychology 60(1):137. DOI: https://doi.org/10.1037/a0030893.

24. Fairburn C G and Beglin S J (2008) Eating Disorder Examination Questionnaire, in Cognitive Behavior Therapy and Eating Disorders. p. 309-313.

25. Kelly N R, Cotter E W, Lydecker J A, and Mazzeo S E (2017) Missing and discrepant data on the Eating Disorder Examination Questionnaire (EDE-Q): Quantity, quality, and implications. Eating Behaviors 24:1-6. DOI:

https://doi.org/10.1016/j.eatbeh.2016.11.002.

26. Ferreira C, Trindade I A, Duarte C, and Pinto-Gouveia J (2015) Getting entangled with body image: Development and validation of a new measure. Psychology and Psychotherapy: Theory, Research and Practice 88(3):304-316. DOI:

https://doi.org/10.1111/papt.12047.

27. Barney J L, Barrett T S, Lensegrav-Benson T, Quakenbush B, and Twohig M P (2021) Confirmatory factor analysis and measurement invariance of the Cognitive Fusion Questionnaire-Body Image in a clinical eating disorder sample. Body Image 38:262-269. DOI: https://doi.org/10.1016/j.bodyim.2021.04.012.

28. Beck A T, Steer R A, and Brown G (1996) Beck depression inventory-II. Psychological Assessment. 
29. Wang Y-P and Gorenstein C (2013) Psychometric properties of the Beck Depression Inventory-II: a comprehensive review. Brazilian Journal of Psychiatry 35(4):416-431. DOI: https://doi.org/10.1590/1516-4446-2012-1048.

30. Udo T, McKee S A, and Grilo C M (2015) Factor structure and clinical utility of the Beck Depression Inventory in patients with binge eating disorder and obesity. General Hospital Psychiatry 37(2):120-125. DOI: https://doi.org/10.1016/j.genhosppsych.2014.11.011.

31. Beck A T, Epstein N, Brown G, and Steer R A (1988) An inventory for measuring clinical anxiety: psychometric properties. Journal of Consulting and Clinical Psychology 56(6):893. DOI: https://doi.org/10.1037/0022-006X.56.6.893.

32. Fydrich T, Dowdall D, and Chambless D L (1992) Reliability and validity of the Beck Anxiety Inventory. Journal of Anxiety Disorders 6(1):55-61. DOI: https://doi.org/10.1016/0887-6185(92)90026-4.

33. R Core Team (2019) R: A language and environment for statistical computing. Vienna, Austria: R Foundation for Statistical Computing.

34. RStudio Team. RStudio: Integrated Development for R. 2019; Available from: http://www.rstudio.com/.

35. Revelle W and Revelle M W (2015) Package 'psych'. The Comprehensive R Archive Network.

36. Selker R, Love J, and Dropmann D, jmv: The 'jamovi' Analyses. 2020.

37. Rosseel Y (2012) lavaan: An R Package for Structural Equation Modeling. Journal of Statistical Software 48(2):1-36. 
38. Cham H, Reshetnyak E, Rosenfeld B, and Breitbart W (2017) Full information maximum likelihood estimation for latent variable interactions with incomplete indicators. Multivariate behavioral research 52(1):12-30. DOI: https://doi.org/10.1080/00273171.2016.1245600.

39. Raykos B C, McEvoy P M, Carter O, Fursland A, and Nathan P (2014) Interpersonal problems across restrictive and binge-purge samples: Data from a community-based eating disorders clinic. Eating behaviors 15(3):449-452. DOI:

https://doi.org/10.1016/j.eatbeh.2014.06.008.

40. Duarte C, Gouveia J P, and Mendes A (2016) Psychometric properties of the Intuitive Eating Scale-2 and association with binge eating symptoms in a Portuguese community sample. International Journal of Psychology and Psychological Therapy 16(3):329-341.

41. Meule A, Richard A, Schnepper R, et al. (2021) Emotion regulation and emotional eating in anorexia nervosa and bulimia nervosa. Eating Disorders 29(2):175-191. DOI: https://doi.org/10.1080/10640266.2019.1642036.

42. Ricca V, Castellini G, Fioravanti G, et al. (2012) Emotional eating in anorexia nervosa and bulimia nervosa. Comprehensive Psychiatry 53(3):245-251. DOI: https://doi.org/10.1016/j.comppsych.2011.04.062.

43. Segura-Garcia C, Ramacciotti C, Rania M, et al. (2015) The prevalence of orthorexia nervosa among eating disorder patients after treatment. Eating and Weight DisordersStudies on Anorexia, Bulimia and Obesity 20(2):161-166. DOI: https://doi.org/10.1007/s40519-014-0171-y. 
44. Bartel S, Sherry S, Farthing G, and Stewart S (2020) Classification of orthorexia nervosa: Further evidence for placement within the eating disorders spectrum. Eating Behaviors 38. DOI: https://doi.org/10.1016/j.eatbeh.2020.101406.

45. Keel P K, Baxter M G, Heatherton T F, and Joiner Jr T E (2007) A 20-year longitudinal study of body weight, dieting, and eating disorder symptoms. Journal of abnormal psychology 116(2):422-432. DOI: https://doi.org/10.1037/0021-843X.116.2.422.

46. Stewart T M, Williamson D A, and White M A (2002) Rigid vs. flexible dieting: association with eating disorder symptoms in nonobese women. Appetite 38(1):39-44. DOI: https://doi.org/10.1006/appe.2001.0445.

47. Fogelkvist M, Gustafsson S A, Kjellin L, and Parling T (2020) Acceptance and commitment therapy to reduce eating disorder symptoms and body image problems in patients with residual eating disorder symptoms: A randomized controlled trial. Body Image 32:155-166. DOI: https://doi.org/10.1016/j.bodyim.2020.01.002.

48. Juarascio A, Shaw J, Forman E, et al. (2013) Acceptance and commitment therapy as a novel treatment for eating disorders: an initial test of efficacy and mediation. Behavior Modification 37(4):459-489. DOI: $\underline{\text { https://doi.org/10.1177/0145445513478633. }}$

49. Luoma J B, Hayes S C, and Walser R D (2007) Learning ACT: An acceptance \& commitment therapy skills-training manual for therapists. Oakland, CA: New Harbinger Publications. 